\title{
Management of flat epithelial atypia on breast core biopsy may be individualized based on correlation with imaging studies
}

\author{
Benjamin C Calhoun ${ }^{1}$, Amy Sobel ${ }^{2}$, Richard L White ${ }^{3}$, Matt Gromet ${ }^{2}$, Teresa Flippo ${ }^{3}$, \\ Terry Sarantou ${ }^{3}$ and Chad A Livasy ${ }^{1,4}$ \\ ${ }^{1}$ Carolinas Pathology Group, Levine Cancer Institute, Charlotte, NC, USA; ${ }^{2}$ Charlotte Radiology, Carolinas \\ Medical Center, Charlotte, NC, USA; ${ }^{3}$ Department of Surgery, Division of Surgical Oncology, Levine Cancer \\ Institute, Charlotte, NC, USA and ${ }^{4}$ Department of Pathology and Laboratory Medicine, University of North \\ Carolina at Chapel Hill, Chapel Hill, NC, USA
}

\begin{abstract}
Flat epithelial atypia of the breast commonly co-exists with atypical ductal hyperplasia, lobular neoplasia, and indolent forms of invasive carcinomas such as tubular carcinoma. Most patients with pure flat epithelial atypia on core biopsy undergo surgical excision to evaluate for carcinoma in the adjacent breast tissue. Studies to date have reported varying upgrade rates with most recommending follow-up excision. These studies have often lacked detailed radiographic correlation, central review by breast pathologists and information regarding the biology of the carcinomas identified upon excision. In this study, we report the frequency of upgrade to invasive carcinoma or ductal carcinoma in situ in excision specimens following a diagnosis of pure flat epithelial atypia on core biopsy. Radiographic correlation is performed for each case and grade/receptor status of detected carcinomas is reported. Seventy-three (73) core biopsies containing pure flat epithelial atypia were identified from our files, meeting inclusion criteria for the study. In the subsequent excision biopsies, five (7\%) cases contained invasive carcinoma or ductal carcinoma in situ and seventeen $(23 \%)$ contained atypical ductal hyperplasia or lobular neoplasia. All of the ductal carcinoma in situ cases with estrogen receptor results were estrogen receptor positive and intermediate grade. The invasive tumors were small (pT1a) hormone receptorpositive, HER2-negative, low-grade invasive ductal or tubular carcinomas with negative sentinel lymph-node biopsies. No upgrades were identified in the 14 patients who had all of their calcifications removed by the stereotactic core biopsy. Our rate of upgrade to carcinoma, once cases with discordant imaging are excluded, is at the lower end of the range reported in the literature. Given the low upgrade rate and indolent nature of the carcinomas associated with flat epithelial atypia, case management may be individualized based on clinical and radiographic findings. Excision may not be necessary for patients without remaining calcifications following core biopsy.
\end{abstract}

Modern Pathology (2015) 28, 670-676; doi:10.1038/modpathol.2014.159; published online 21 November 2014

Columnar cell lesions of the breast are common findings in core biopsies performed to evaluate mammographically indeterminate calcifications. Columnar cell lesions with nuclear atypia are classified as flat epithelial atypia by the World Health Organization (WHO)., ${ }^{1,2}$ The management of

Correspondence: Dr BC Calhoun, MD, PhD, Department of Pathology, Cleveland Clinic Foundation,, 9500 Euclid Avenue, Mail Code L25, Cleveland, OH 44195, USA.

E-mail: calhoub@ccf.org

Preliminary results were presented at the San Antonio Breast Cancer Symposium in 2013.

Received 7 September 2014; revised 15 October 2014; accepted 16 October 2014; published online 21 November 2014 flat epithelial atypia diagnosed on core biopsy remains controversial. ${ }^{3}$ However, many patients with flat epithelial atypia are offered local excision to evaluate the possibility of a co-existing carcinoma. In a recent meta-analysis of flat epithelial atypia core biopsy studies, carcinoma was present in excisional biopsies in $13-67 \%$ of cases. ${ }^{4}$

Columnar cell lesions and flat epithelial atypia have been recognized for many years and called by many different names. ${ }^{5}$ Columnar cell lesions have been referred to as 'blunt duct adenosis', ${ }^{6-8}$ 'hyperplastic enlarged lobular units', 9 'columnar alteration with prominent apical snouts and secretions', 10 and 'enlarged lobular units with columnar alteration'. ${ }^{11}$ Flat epithelial atypia has been referred 
to as 'clinging carcinoma, monomorphic type', ${ }^{12}$ ductal intraepithelial neoplasia, ${ }^{13}$ and columnar cell change or columnar cell hyperplasia with atypia. $^{14}$ The current WHO classification recognizes three diagnostic categories: columnar cell change, columnar cell hyperplasia, and flat epithelial atypia. ${ }^{2}$ In this classification, columnar cell change and columnar cell hyperplasia with atypia are classified as flat epithelial atypia.

Several lines of evidence suggest a potential relationship between flat epithelial atypia and breast neoplasia. ${ }^{15}$ Columnar cell lesions and flat epithelial atypia are often co-localized with other patterns of atypia such as atypical ductal hyperplasia and atypical lobular hyperplasia ${ }^{16,17}$ and low-grade in situ and invasive carcinomas. ${ }^{18}$ Molecular studies have shown similar genetic alterations in flat epithelial atypia, atypical hyperplasia, and low-grade carcinoma in the same tissue section, suggesting that flat epithelial atypia may be a non-obligate precursor in a low-grade breast neoplasia pathway. ${ }^{19,20}$ In long-term follow-up studies of open biopsies, the risk associated with columnar cell lesions and flat epithelial atypia alone appears to be lower than the risk associated with atypical ductal or atypical lobular hyperplasia. ${ }^{16,17}$

In a prior series from our institution, we reported a series of 31 cases from 1998 to 2003, with available follow-up excisions, 4 (12.9\%) of which contained carcinoma. ${ }^{21}$ That study predated the WHO classification of flat epithelial atypia, ${ }^{1}$ and we now report the frequency of carcinoma in excision specimens after a diagnosis of flat epithelial atypia on core biopsy for a subsequent 9-year period (2004-2013).

\section{Materials and methods}

The study was approved the Institutional Review Board of Carolinas Medical Center. Breast core biopsies with a diagnosis of flat epithelial atypia from 1 January 2004 to 15 October 2013 were retrieved from the Anatomic Pathology information system (CoPath). For all core biopsies, the presence and type of co-existing atypical ductal hyperplasia or atypical lobular hyperplasia or carcinoma was recorded. Cases with co-existing atypical ductal hyperplasia or atypical lobular hyperplasia or carcinoma and cases without an excision pathology report in CoPath were excluded from further study. Cases with atypical ductal hyperplasia or atypical lobular hyperplasia, carcinoma in situ, or invasive carcinoma in ipsilateral core biopsies performed concurrently or within 4 months of the core biopsy with flat epithelial atypia were also excluded. All study cases were reviewed by dedicated breast pathologists (CAL and BCC) to confirm the diagnosis of pure flat epithelial atypia using WHO criteria. These criteria include enlarged terminal duct lobular units, often with 3-5 cell layers, with proliferation of cells showing low-grade monomorphic cytology, increased nuclear/cytoplasmic ratios, and loss of polarity (Figure 1). Multiple levels were examined in all cases. From 2009 through completion of the study period, a cutting protocol obtaining six (6) H\&E levels 150-250 microns apart were routinely examined for each stereotactic core biopsy. Before that change, the diagnostic pathologist requested additional levels (usually three) in all cases in which a 'stand alone' diagnosis of flat epithelial atypia was entertained. At least six levels were examined in the final study group (73 core biopsies with flat epithelial atypia alone).

All available surgical pathology reports for excisions following a core biopsy diagnosis of flat epithelial atypia alone were reviewed. Excision specimens with ductal carcinoma in situ or invasive carcinoma were also reviewed by breast pathologists (BCC and CAL) to confirm diagnosis. Three patients had two sites with flat epithelial atypia alone on core biopsy, and each core biopsy site was individually correlated with an excision specimen. The presence and location of calcifications was included in the pathology reports for the core biopsies and the excision specimens. It was not routine practice to submit the entire surgical specimen for large excisions (>15-20 blocks) following a core biopsy diagnosis of atypia. Cases were thoroughly sampled to include the prior biopsy site region and any remaining calcifications within the specimen through correlation with localization needle, biopsy clip(s), and calcifications identified on accompanying specimen image or Faxitron image in our gross room.

The indication for core biopsy, BIRADS score, percentage of calcifications removed, and concordance with histology were recorded by dedicated breast radiologists. Cases were excluded from the study when the overall imaging findings were interpreted by the breast radiologist as discordant with the pathologic diagnosis of flat epithelial atypia. This includes one case with MRI findings of multiple areas of clumped segmental enhancement highly suspicious for ductal carcinoma in situ. As the primary objective of this study was to establish the rate of upgrade to carcinoma, only cases with concordant imaging and carcinoma (invasive ductal or lobular carcinoma or ductal carcinoma in situ on excision) were regarded as pathologically upgraded. The presence of other riskassociated histologic findings including atypical ductal hyperplasia, atypical lobular hyperplasia, and lobular carcinoma in situ was also recorded. The vacuum-assisted stereotactic core biopsies were performed with nine gauge ATEC or EVIVA devices. The ultrasound core biopsies were performed with a 14gauge BARD reusable spring-loaded device, and the vacuum-assisted MRI-guided core biopsies were performed with a 9-gauge ATEC device.

\section{Results}

A total of 22707 breast core biopsies were performed during the study period with a mean and median of 

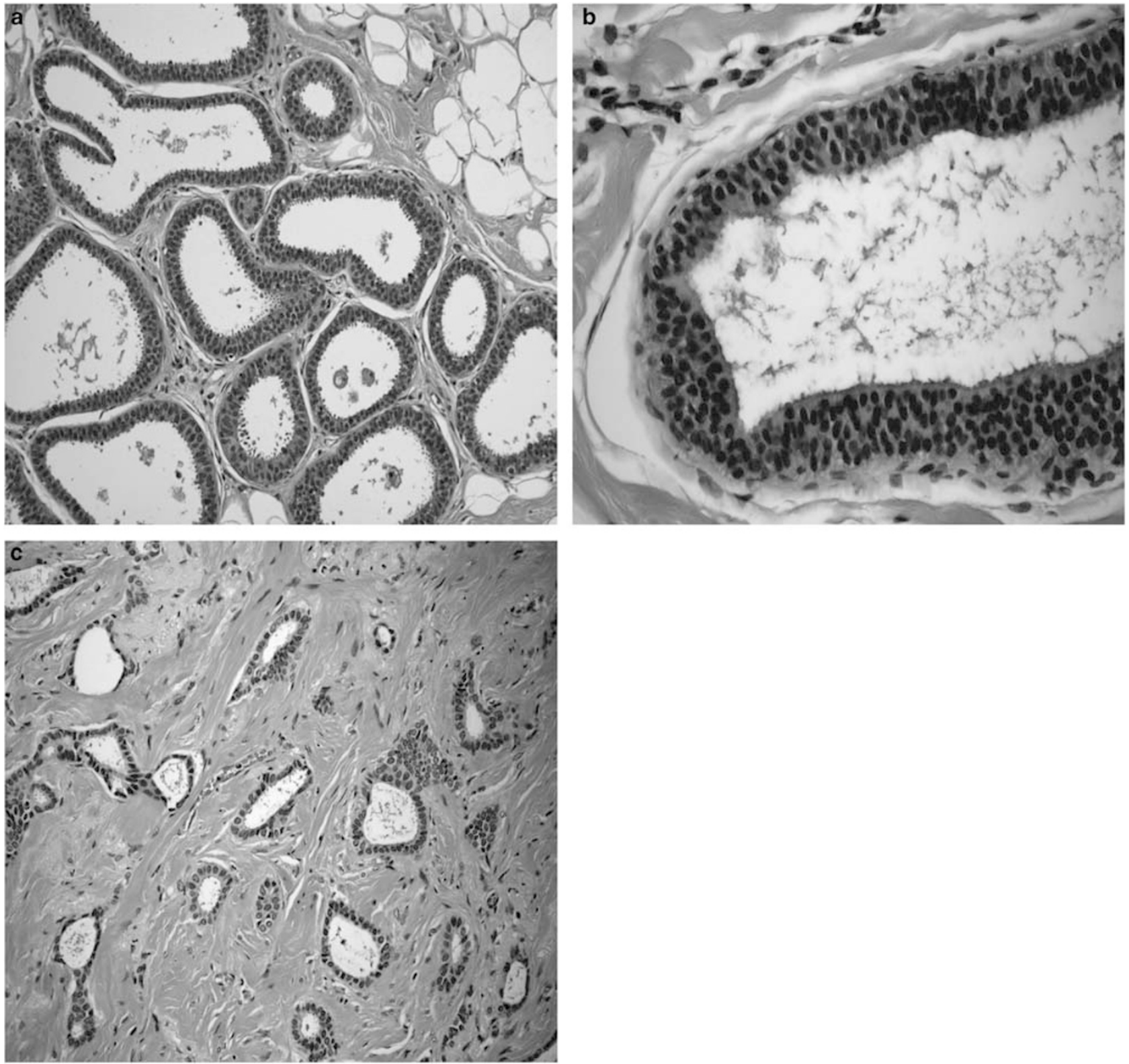

Figure 1 Representative images illustrating the criteria used for the diagnosis of flat epithelial atypia (a and $\mathbf{b}$, at $\times 200$ and $\times 400$ magnification, respectively) and tubular carcinoma (c, at $\times 200$ magnification).

2305 and 2219 per year, respectively. Two hundredten (210) core biopsies from 201 patients were identified as containing flat epithelial atypia (Table 1). Two (2) cases were ultrasound core biopsies, 5 were MRI-guided, and the remaining 203 were stereotactic core biopsies for calcifications. Radiology data were incomplete for three cases, none of which were upgraded. One of the mammograms preceding core biopsy was BIRADS 3 and the remaining cases BIRADS 4. In 116 biopsies, a higher risk form of atypia or carcinoma was present: 60 (29\%) contained atypical ductal hyperplasia, 19 (9\%) atypical lobular hyperplasia, 9 (4\%) atypical ductal hyperplasia and atypical lobular hyperplasia,
$14(7 \%)$ ductal carcinoma in situ, 2 (1\%) lobular carcinoma in situ, and $12(6 \%)$ invasive carcinoma. In the cores with invasive carcinoma, eight were tubular carcinomas (or tubular features), three were low- or intermediate-grade invasive ductal carcinoma, no special type (grades 4/9, 5/9, 6/9), and one was microinvasion (less than $1 \mathrm{~mm}$ ) associated ductal carcinoma in situ. These cases were excluded from further analysis.

Flat epithelial atypia was the most advanced lesion in $94(45 \%)$ core biopsies from 90 patients with a median age at the time of core biopsy of 47 (range 33-71). Fifty (50) patients were premenopausal, 24 were postmenopausal (or had undergone 
Table 1 Flat epithelial atypia and co-existing lesions on core biopsy

\begin{tabular}{cccccccc}
\hline Total & FEA only & ADH & ALH & ADH \& ALH & LCIS & DCIS & Invasive \\
\hline 210 & $94(45 \%)^{\mathrm{a}}$ & $60(29 \%)$ & $19(9 \%)$ & $9(4 \%)$ & $2(1 \%)$ & $14(7 \%)$ & $12(6 \%)$
\end{tabular}

Abbreviations: ADH, atypical ductal hyperplasia; ALH, atypical lobular hyperplasia; DCIS, ductal carcinoma in situ; FEA, flat epithelial atypia; Invasive, invasive carcinoma; LCIS, lobular carcinoma in situ.

Two hundred-ten (210) core biopsies with flat epithelial atypia from 201 patients were identified from 2004 to 2013 . Of those, 116 contained a higher risk form of atypia or carcinoma and were excluded from further analysis. In the cores with invasive carcinoma, 8 were tubular carcinomas (or tubular features), 3 were low- or intermediate-grade invasive ductal carcinoma, no special type (grades 4/9, 5/9, 6/9), and 1 was microinvasion (less than $1 \mathrm{~mm}$ ) associated DCIS.

apercentages rounded to nearest integer.

Table 2 Excision after flat epithelial atypia alone on core biopsy

\begin{tabular}{lcccccc}
\hline Total & No atypia & FEA & ADH & ALH & DCIS & Invasive \\
\hline 73 & $20(27 \%)^{\mathrm{a}}$ & $31(42 \%)$ & $14(19 \%)$ & $3(4 \%)$ & $3(4 \%)$ & $2(3 \%)$ \\
\hline
\end{tabular}

Abbreviations: ADH, atypical ductal hyperplasia; ALH, atypical lobular hyperplasia; DCIS, ductal carcinoma in situ; FEA, flat epithelial atypia; Invasive, invasive carcinoma.

Of the 94 core biopsies with flat epithelial atypia alone, 21 were excluded from further study. The overall rate of upgrade to carcinoma for the remaining 73 core biopsies was 7\% (5/73). Ductal carcinoma in situ and a low-grade invasive ductal carcinoma, no special type, were present in 3 $(4 \%)$ and $2(3 \%)$ excision specimens, respectively. Seventeen excision specimens $(23 \%)$ contained another, higher risk pattern of atypia (atypical ductal or atypical lobular hyperplasia).

aPercentages rounded to nearest integer.

Table 3 Flat epithelial atypia alone on core biopsy with carcinoma on excision

\begin{tabular}{|c|c|c|c|c|c|c|}
\hline Diagnosis & Size $(\mathrm{mm})$ & Grade & Estrogen receptor & $B I R A D S$ & Indication & Calcs removed \\
\hline IDC (NST) & 3 & 1 & Positive & 4 & $10 \mathrm{~mm}$ calcs & $<25 \%$ \\
\hline IDC (tubular) & 3 & 1 & Positive & 4 & 6 mm calcs & $>75 \%$ \\
\hline DCIS & 8 & 2 & Positive & 4 & $12 \mathrm{~mm}$ calcs & $>75 \%$ \\
\hline DCIS & 52 & 2 & NR & 4 & $40 \mathrm{~mm}$ calcs & $<25 \%$ \\
\hline DCIS & 38 & 2 & Positive & 4 & $23 \mathrm{~mm}$ density & N/A \\
\hline
\end{tabular}

Abbreviations: Calcs, calcifications; DCIS, ductal carcinoma in situ; IDC, invasive ductal carcinoma; NST, no special type.

Of the five cases upgraded to carcinoma, two were invasive carcinomas measuring $3 \mathrm{~mm}$ each and three were intermediate-grade ductal carcinoma in situ. The two cases of ductal carcinoma in situ with available estrogen receptor immunohistochemistry results were estrogen receptor positive.

hysterectomy), 3 were peri-menopausal, and menopausal status was unknown/unrecorded from 13 patients. Seventeen (17) patients had a first-degree relative with a history of breast cancer, 14 patients had a second- or third-degree relative with breast cancer, and 41 patients had no family history of breast cancer. Family history was unknown/unrecorded for 18 patients. Six (6) patients had a personal history of prior invasive carcinoma or ductal carcinoma in situ and 5 patients had a prior diagnosis of atypia (3 atypical lobular hyperplasia and 2 flat epithelial atypia).

Of the 94 core biopsies with flat epithelial atypia alone, 21 were excluded from further study due to excision pathology reports being unavailable for 12 cases, an ipsilateral core biopsy containing a more advanced lesion in 8 cases, and one case with discordant imaging findings. On retrospective review for this study, the discordant case was classified as a BIRADS-5 imaging study that would have required open biopsy regardless of the core biopsy findings. The overall rate of upgrade to carcinoma for the remaining 73 core biopsies that comprised the final study group was $7 \%(5 / 73)$ (Table 2). Ductal carcinoma in situ and a low-grade invasive ductal carcinoma, no special type, were present in $3(4 \%)$ and $2(3 \%)$ excision specimens, respectively. The most common finding on excision was FEA alone $(43 \%)$. Fifty-one $(70 \%)$ specimens contained either flat epithelial atypia or no atypia and seventeen $(23 \%)$ contained another, higher risk pattern of atypia (atypical ductal or atypical lobular hyperplasia). None of the 14 cases with all of the calcifications removed by the stereotactic core biopsy was upgraded to carcinoma.

The features of the cases upgraded to carcinoma are summarized in Table 3. The two invasive carcinomas measured $3 \mathrm{~mm}$ each and were positive 
Table 4 Selected studies: flat epithelial atypia alone on core biopsy with excision

\begin{tabular}{|c|c|c|c|c|c|c|}
\hline & Core biopsies & Excisions & Carcinoma (\%) & $D C I S$ & Invasive & Recommendation \\
\hline Lavoue et $a l^{38}$ & 60 & 60 & $8(13 \%)^{\mathrm{a}}$ & 6 & 2 & Excision \\
\hline Guerra-Wallace et $a 2^{21}$ & 39 & 31 & $4(13 \%)$ & 3 & 1 & Excision \\
\hline Bianchi et $a l^{27}$ & 190 & 190 & $18(10 \%)$ & NR & NR & Excision \\
\hline Chivukula et $a l^{33}$ & 39 & 35 & $5(14 \%)$ & 3 & 2 & Excision \\
\hline Noske et $a l^{39}$ & 43 & 30 & $2(7 \%)$ & 2 & 0 & Excision \\
\hline Senetta et $a l^{40}$ & 41 & 36 & $0(0 \%)$ & 0 & 0 & Case by case \\
\hline Ceugnart et al ${ }^{41}$ & 63 & 52 & $2(4 \%)$ & 2 & 0 & Case by case \\
\hline Biggar et $a l^{42}$ & 51 & 51 & $3(6 \%)$ & 2 & 1 & Excision \\
\hline Khoumais et $a l^{43}$ & 104 & 94 & $10(11 \%)$ & 5 & 5 & Excision \\
\hline Uzoaru et $a l^{44}$ & 145 & 95 & $3(3 \%)$ & 1 & 2 & Case by case \\
\hline Peres et $a l^{45}$ & 128 & 95 & $9(10 \%)$ & 5 & 4 & Excision \\
\hline Villa et $a 4^{46}$ & 142 & 121 & $7(6 \%)$ & NR & NR & Case by case \\
\hline This study & 94 & 73 & $5(7 \%)$ & 3 & 2 & Case by case \\
\hline Total & 1139 & 963 & $76(8 \%)$ & 32 & 19 & \\
\hline
\end{tabular}

Abbreviations: DCIS, ductal carcinoma in situ; Invasive, invasive carcinoma; NR, not reported.

Comparison of upgrade rates in selected studies of flat epithelial atypia on core biopsy (adapted from Verschuur-Maes et al ${ }^{4}$ to include studies with 30 or more excisions and to include studies from 2010 to 2013).

apercentages rounded to nearest integer.

for estrogen receptor and negative for HER-2 gene amplification or protein overexpression. Subsequent axillary sentinel lymph-node biopsies from both patients were negative for tumor. The three cases upgraded to ductal carcinoma in situ were all intermediate nuclear grade (2/3) and measured 8, 38 , and $52 \mathrm{~mm}$. In the case with $52 \mathrm{~mm}$ of ductal carcinoma in situ, less than $25 \%$ of calcifications were removed at the time of core biopsy. The two cases of ductal carcinoma in situ with available estrogen receptor immunohistochemistry results were estrogen receptor positive. One of the patients upgraded to ductal carcinoma in situ had a firstdegree relative with a history of breast cancer. None of the other patients upgraded to carcinoma had a family history of breast cancer. The indication for biopsy in the case with $38 \mathrm{~mm}$ of ductal carcinoma in situ was an asymmetric density. Calcifications were the indication for the core biopsies in all of the other upgraded cases.

\section{Discussion}

This is one of the larger retrospective series of core biopsies with flat epithelial atypia alone from a single institution. In a recent meta-analysis of 22 studies published between 1990 and 2010, the frequency of finding carcinoma in an excision performed for flat epithelial atypia on a core ranged from 13 to $67 \% .{ }^{4}$ Determining a clinically relevant upgrade rate requires correlation of radiology and pathology findings to exclude discordant cases. However, the radiology-pathology correlation data are either missing or incomplete in many studies. ${ }^{4}$ Studies that have included radiology-pathology correlation for core biopsy cases with papillomas ${ }^{22,23}$ and lobular neoplasia $^{24-26}$ tend to report lower upgrade rates and may argue against mandatory excision for these diagnoses on core biopsy. Similarly, our overall upgrade rate of
$7 \%$ for cases with careful correlation is lower than the majority of published studies with 30 or more excisions (Table 4) and closer to the $9.5 \%$ reported by Bianchi et $a l^{27}$ in a series of 589 cases from multiple hospitals in Italy. Another recent study of 24 cases showed an upgrade rate of $3.2 \% .{ }^{28}$ Piubello et $a 1^{29}$ and Noel et al ${ }^{30}$ reported no cases with carcinoma on excision in two smaller series in which only the patients with residual suspicious calcifications underwent surgical biopsy for flat epithelial atypia. It must also be noted that all but one of the pre-core biopsy imaging studies in our series were BIRADS-4. All of the cases reported by Noel et $a l^{30}$ and all but two of the cases reported by Piubello et $a 1^{29}$ were BIRADS-3.

The limitations of this study include potential interobserver variability in the diagnosis of flat epithelial atypia among pathologists, ${ }^{31}$ a change in our microtomy protocol during the study period, the potential for sampling error in excisions that were not entirely submitted for histologic examination, and the variability in the needle gauge used for core biopsy. With the exception of the studies by Kunju and $\mathrm{Kleer}^{32}$ and Chivukula et $a,^{33}$ the data on the histologic sampling of the core biopsies with flat epithelial atypia are limited, and many studies are based on the routine examination of three (3) H\&E levels at potentially varying depths in the tissue block. The data on gross sampling of excision specimens are similarly limited. In our practice, we may not entirely submit large lumpectomies for atypical hyperplasias and flat epithelial atypia diagnosed on core biopsy, but the entire biopsy site and all adjacent breast tissue are submitted.

The primary goal in the clinical management of patients with epithelial atypia of the breast arguably should be risk reduction rather than detection of other potential cancer precursors or small, indolent tumors. But, two different categories of risk must be considered. In the pre-core biopsy and pre-mammo- 
graphic eras, the risk associated with atypical ductal hyperplasia and atypical lobular hyperplasia defined in long-term follow-up studies was defined as the relative risk (or odds ratio) of the patient developing a subsequent carcinoma (in either breast), usually 10-15 years after an open biopsy. ${ }^{34,35}$ As percutaneous breast core biopsy became more routine, the risk of finding carcinoma in tissue surrounding the core biopsy site became a more immediate concern. For patients with a core biopsy diagnosis of flat epithelial atypia, referral to a surgeon and the decision to excise an area of flat epithelial atypia are more likely based on the risk of finding carcinoma in adjacent tissue, rather the long-term relative risk of developing a carcinoma in the future in either breast. However, to the best of our knowledge there have been no prospective trials randomizing patients with flat epithelial atypia or any other pattern of atypia to excision vs close observation. Although the rate of upgrade to carcinoma in our series is quite low, the $23 \%$ of excision specimens with atypical ductal hyperplasia or atypical lobular hyperplasia in this study could have implications for risk stratification and decisions about chemoprevention.

The invasive carcinomas detected in this study were small, low grade, and hormone receptor positive. Two of the three cases upgraded to ductal carcinoma in situ with available hormone receptor results were estrogen receptor positive and none was high grade. The detection of these tumors may represent examples of overdiagnosis. ${ }^{36,37}$ And, if these patients had undergone surveillance rather than surgery, the clinically relevant tumors could likely have been identified at a later date at a point at which they would still be curable. In the two cases with 38 and $52 \mathrm{~mm}$ of ductal carcinoma in situ, less than $50 \%$ of the mammographic abnormalities were removed by core biopsy. None of the 14 cases with all of the calcifications removed by the stereotactic core biopsy was upgraded to carcinoma. These findings suggest that radiographic correlation may help in stratifying risk for upgrade. Patients with complete removal of targeted calcifications at biopsy may choose to undergo close surveillance rather than excision due to the very low risk of upgrading to carcinoma. In contrast, it seems prudent to offer excision to patients with a core biopsy diagnosis of flat epithelial atypia with limited sampling of the radiographic target or discordant imaging findings, similar to what has been proposed for solitary papillomas and lobular neoplasia diagnosed on core biopsy. ${ }^{24-26}$

Most studies evaluating the upgrade rates of flat epithelial atypia on core biopsy have not reported the characteristics of the carcinoma upgrades identified on excision. As this study demonstrates, flat epithelial atypia is associated with other forms of low-grade atypia and low-grade estrogen receptorpositive carcinomas. The carcinomas found on excision in this study were pT1a, low-grade estrogen receptor-positive invasive carcinomas or estrogen receptor-positive ductal carcinoma in situ. In an era of increasing recognition of overdiagnosis and overtreatment of breast lesions detected by screening mammography, the clinical value of detecting the upgrades in these patients could be challenged. ${ }^{36,37}$

\section{Disclosure/conflict of interest}

The authors declare no conflict of interest.

\section{References}

1 Tavassoli FA, Hoefler H, Rosai J, et al. Pathology and genetics of tumors of the breast and female genital organs. In: Tavassoli FA, Devilee P (eds). World Health Organization Classification of Tumours, 3rd edn. International Agency for Research on Cancer (IARC): Lyon, France; 2003, pp 65-66.

2 Schnitt SJ, Collins L, Lakhani SR, et al. Flat epithelial atypia. In: Lakhani SR, Ellis IO, Schnitt SJ, Tan PH, van de Vijver MJ (eds). World Health Organization Classification of Tumours, 4th edn. International Agency for Research on Cancer (IARC): Lyon, France, 2012, p 87.

3 Georgian-Smith D, Lawton TJ. Variations in physician recommendations for surgery after diagnosis of a highrisk lesion on breast core needle biopsy. AJR Am J Roentgenol 2012;198:256-263.

4 Verschuur-Maes AH, van Deurzen CH, Monninkhof EM, et al. Columnar cell lesions on breast needle biopsies: is surgical excision necessary? A systematic review. Ann Surg 2012;255:259-265.

5 Schnitt SJ, Collins LC. Columnar cell lesions and flat epithelial atypia of the breast. Semin Breast Dis 2005;8:100-111.

6 Foote FW, Stewart FW. Comparative studies of cancerous versus noncancerous breasts. Ann Surg 1945;121:197-222.

7 Haagensen CD. Diseases of the Breast, 2nd edn. W.B. Saunders Company: Philadelphia, PA, 1971.

8 Rosen PP, Oberman HA. Tumors of the Mammary Gland. Rosai J, Sobin L (eds). Armed Forces Institute of Pathology: Washington, DC, 1993.

9 Lee S, Mohsin SK, Mao S, et al. Hormones, receptors, and growth in hyperplastic enlarged lobular units: early potential precursors of breast cancer. Breast Cancer Res 2006;8:R6.

10 Fraser JL, Raza S, Chorny K, et al. Columnar alteration with prominent apical snouts and secretions: a spectrum of changes frequently present in breast biopsies performed for microcalcifications. Am J Surg Pathol 1998;22:1521-1527.

11 McLaren BK, Gobbi H, Schuyler PA, et al. Immunohistochemical expression of estrogen receptor in enlarged lobular units with columnar alteration in benign breast biopsies: a nested case-control study. Am J Surg Pathol 2005;29:105-108.

12 Azzopardi JG, Ahmed A, Millis RR. Clinging carcinoma. In: Bennington JL (ed). Major Problems in Breast Pathology. W.B. Saunders Company Ltd.: Philadelphia, PA; 1979, pp 193-203.

13 Tavassoli FA. Breast pathology: rationale for adopting the ductal intraepithelial neoplasia (DIN) classification. Nat Clin Pract Oncol 2005;2:116-117. 
14 Schnitt SJ, Vincent-Salomon A. Columnar cell lesions of the breast. Adv Anat Pathol 2003;10:113-124.

15 Lopez-Garcia MA, Geyer FC, Lacroix-Triki M, et al. Breast cancer precursors revisited: molecular features and progression pathways. Histopathology 2010;57: 171-192.

16 Boulos FI, Dupont WD, Simpson JF, et al. Histologic associations and long-term cancer risk in columnar cell lesions of the breast: a retrospective cohort and a nested case-control study. Cancer 2008;113:2415-2421.

17 Aroner SA, Collins LC, Schnitt SJ, et al. Columnar cell lesions and subsequent breast cancer risk: a nested case-control study. Breast Cancer Res 2010;12:R61.

18 Abdel-Fatah TM, Powe DG, Hodi Z, et al. High frequency of coexistence of columnar cell lesions, lobular neoplasia, and low grade ductal carcinoma in situ with invasive tubular carcinoma and invasive lobular carcinoma. Am J Surg Pathol 2007;31:417-426.

19 Abdel-Fatah TM, Powe DG, Hodi Z, et al. Morphologic and molecular evolutionary pathways of low nuclear grade invasive breast cancers and their putative precursor lesions: further evidence to support the concept of low nuclear grade breast neoplasia family. Am J Surg Pathol 2008;32:513-523.

20 Simpson PT, Gale T, Reis-Filho JS, et al. Columnar cell lesions of the breast: the missing link in breast cancer progression? A morphological and molecular analysis. Am J Surg Pathol 2005;29:734-746.

21 Guerra-Wallace MM, Christensen WN, White RL Jr. A retrospective study of columnar alteration with prominent apical snouts and secretions and the association with cancer. Am J Surg 2004;188:395-398.

22 Ivan D, Selinko V, Sahin AA, et al. Accuracy of core needle biopsy diagnosis in assessing papillary breast lesions: histologic predictors of malignancy. Mod Pathol 2004;17:165-171.

23 Swapp RE, Glazebrook KN, Jones KN, et al. Management of benign intraductal solitary papilloma diagnosed on core needle biopsy. Ann Surg Oncol 2013;20:1900-1905.

24 Middleton LP, Grant S, Stephens T, et al. Lobular carcinoma in situ diagnosed by core needle biopsy: when should it be excised? Mod Pathol 2003;16: 120-129.

25 Rendi MH, Dintzis SM, Lehman CD, et al. Lobular insitu neoplasia on breast core needle biopsy: imaging indication and pathologic extent can identify which patients require excisional biopsy. Ann Surg Oncol 2012;19:914-921.

26 D’Alfonso TM, Wang K, Chiu YL, et al. Pathologic upgrade rates on subsequent excision when lobular carcinoma in situ is the primary diagnosis in the needle core biopsy with special attention to the radiographic target. Arch Pathol Lab Med 2013;137:927-935.

27 Bianchi S, Bendinelli B, Castellano I, et al. Morphological parameters of flat epithelial atypia (FEA) in stereotactic vacuum-assisted needle core biopsies do not predict the presence of malignancy on subsequent surgical excision. Virchows Arch 2012;461:405-417.

28 Prowler VL, Joh JE, Acs G, et al. Surgical excision of pure flat epithelial atypia identified on core needle breast biopsy. Breast 2014;23:352-356.

29 Piubello Q, Parisi A, Eccher A, et al. Flat epithelial atypia on core needle biopsy: which is the right management? Am J Surg Pathol 2009;33:1078-1084.
30 Noel JC, Buxant F, Engohan-Aloghe C. Immediate surgical resection of residual microcalcifications after a diagnosis of pure flat epithelial atypia on core biopsy: a word of caution. Surg Oncol 2010;19: 243-246.

31 O’Malley FP, Mohsin SK, Badve S, et al. Interobserver reproducibility in the diagnosis of flat epithelial atypia of the breast. Mod Pathol 2006;19:172-179.

32 Kunju LP, Kleer CG. Significance of flat epithelial atypia on mammotome core needle biopsy: Should it be excised? Hum Pathol 2007;38:35-41.

33 Chivukula $\mathrm{M}$, Bhargava $\mathrm{R}$, Tseng $\mathrm{G}$, et al. Clinicopathologic implications of "flat epithelial atypia" in core needle biopsy specimens of the breast. Am J Clin Pathol 2009;131:802-808.

34 Collins LC, Baer HJ, Tamimi RM, et al. Magnitude and laterality of breast cancer risk according to histologic type of atypical hyperplasia: results from the Nurses' Health Study. Cancer 2007;109:180-187.

35 Dupont WD, Page DL. Risk factors for breast cancer in women with proliferative breast disease. N Engl J Med 1985;312:146-151.

36 Calhoun BC, Livasy CA. Mitigating overdiagnosis and overtreatment in breast cancer: what is the role of the pathologist? Arch Pathol Lab Med 2014;138: 1428-1431.

37 Esserman LJ, Thompson IM Jr., Reid B. Overdiagnosis and overtreatment in cancer: an opportunity for improvement. JAMA 2013;310:797-798.

38 Lavoue V, Roger CM, Poilblanc M, et al. Pure flat epithelial atypia (DIN 1a) on core needle biopsy: study of 60 biopsies with follow-up surgical excision. Breast Cancer Res Treat 2011;125:121-126.

39 Noske A, Pahl S, Fallenberg E, et al. Flat epithelial atypia is a common subtype of B3 breast lesions and is associated with noninvasive cancer but not with invasive cancer in final excision histology. Hum Pathol 2010;41:522-527.

40 Senetta R, Campanino PP, Mariscotti G, et al. Columnar cell lesions associated with breast calcifications on vacuum-assisted core biopsies: clinical, radiographic, and histological correlations. Mod Pathol 2009;22:762-769.

41 Ceugnart L, Doualliez V, Chauvet MP, et al. Pure flat epithelial atypia: Is there a place for routine surgery? Diagn Interv Imaging 2013;94:861-869.

42 Biggar MA, Kerr KM, Erzetich LM, et al. Columnar cell change with atypia (flat epithelial atypia) on breast core biopsy-outcomes following open excision. Breast J 2012;18:578-581.

43 Khoumais NA, Scaranelo AM, Moshonov $\mathrm{H}$, et al. Incidence of breast cancer in patients with pure flat epithelial atypia diagnosed at core-needle biopsy of the breast. Ann Surg Oncol 2013;20:133-138.

44 Uzoaru I, Morgan BR, Liu ZG, et al. Flat epithelial atypia with and without atypical ductal hyperplasia: to re-excise or not. Results of a 5-year prospective study. Virchows Arch 2012;461:419-423.

45 Peres A, Barranger E, Becette V, et al. Rates of upgrade to malignancy for 271 cases of flat epithelial atypia (FEA) diagnosed by breast core biopsy. Breast Cancer Res Treat 2012;133:659-666.

46 Villa A, Chiesa F, Massa T, et al. Flat epithelial atypia: comparison between 9-gauge and 11-gauge devices. Clin Breast Cancer 2013;13:450-454. 